\title{
Algorithmic Manifolds and Their Properties
}

\author{
Takuya Yabu(takuya.yabu@live.jp)
}

\section{Introduction}

About computational complexity [1], it has been studied for long time. Recent literature [2] has shown that the existing proof method using the diagonal argument or the circuit complexity is not effective. On the other hand, as another approach, calculation of time complexity based on the geometric method is also performed [3], but it is limited to the quantum algorithm, and it is an application example to the existing method of lower band derivation of quantum circuit complexity, it is essentially unchanged.

In this paper, I introduce algorithmic manifolds that explain algorithms by geometric methods and discuss their properties. In the section 2, I discuss the relationship between algorithmic manifolds and time. In the section 3, I discuss the relationship between algorithmic manifolds and amounts of data. In the section 4, I discuss topological characteristics of algorithmic manifolds. The section 5 is the conclusion of this paper.

\section{Relationship between algorithmic manifolds and time}

Think about the Riemannian manifold defined by the following metric.

$$
d s^{2}=d v^{2}+d \tau^{2}=d v^{2}+\llbracket f(v)^{2} \rrbracket d c^{2}
$$

Where $v \geq 0$ is amounts of data to be processed, $\tau$ is time, $c$ is amounts of calculation at that time, $\llbracket f(v) \rrbracket$ is a function of the time required to process a certain amount of calculation, $\tau=\llbracket f(v) \rrbracket c$. All variables and functions are real numbers or real functions. The negative values of $\tau$ and $c$ mean the past. In addition, $\llbracket f(v) \rrbracket$ means that it is a function focusing on the property of $f(v)$ when $v$ is sufficiently large, and the following relational expression holds. Here, $a, b$ is a real number, and $g(v)$ is a real function.

$$
\begin{gathered}
a \llbracket f(v) \rrbracket=\operatorname{sgn}(a) f(v) \\
\llbracket a f(v) \rrbracket=\operatorname{sgn}(a) f(v) \\
\frac{d}{d v} \llbracket f(v) \rrbracket=\llbracket \frac{d}{d v} f(v) \rrbracket \\
\llbracket f(v) \rrbracket \llbracket g(v) \rrbracket=\llbracket f(v) g(v) \rrbracket \\
\frac{\llbracket f(v) \rrbracket}{\llbracket g(v) \rrbracket}=\llbracket \frac{f(v)}{g(v)} \rrbracket
\end{gathered}
$$




$$
\begin{aligned}
\llbracket a f(v)+b g(v) \rrbracket= & \operatorname{sgn}(a) f(v) \text { at } f(v)>g(v) \text { when } v \text { is } \\
& \text { sufficiently large } \\
a \llbracket f(v) \rrbracket+b \llbracket g(v) \rrbracket= & \operatorname{sgn}(a) f(v) \text { at } f(v)>g(v) \text { when } v \text { is } \\
& \text { sufficiently large }
\end{aligned}
$$

The Riemannian manifold defined by the above metric ( 1 ) is called the algorithmic manifold below. Since the algorithmic manifold is a subset of Euclidean space, it is a complete metric space. Therefore, from the Hopf-Rinow theorem [4], there is a geodesic between arbitrary two points. Also, since the algorithmic manifold is a complete metric space, the Cauchy sequence converges. Therefore, since the algorithmic manifold is complete and totally bounded, it is also compact.

In the equation ( 1$)$, consider the following $f(v)$. Here, $k(c)$ is a real number variable defined so as to satisfy the relational expression of $\tau$ and $c$.

$$
f(v)=v^{k(c)}
$$

The Gaussian curvature $K$ of an algorithmic manifold with a metric consisting of equations (1) and (9) is given by the following equation.

$$
K=\left\{\begin{array}{c}
0(k(c)=0) \\
\frac{1}{v^{2}}\left(0<k(c) \leq \frac{1}{2}\right) \\
0\left(k(c)>\frac{1}{2}\right)
\end{array}\right.
$$

When $k(c)=0$ and $k(c)>\frac{1}{2}$, from equation (10), the algorithmic manifold with the metric consisting of equations (1) and (9) is complete and its Gaussian curvature $K$ is zero everywhere, so the algorithmic manifold is a cylinder from Hartman-Nirenberg theorem [5]. When $0<k(c) \leq \frac{1}{2}$ and $v>0$, from equation ( 10), the algorithmic manifold with the metric consisting of equations ( 1$)$ and (9) is compact and its Gaussian curvature $K$ is positive everywhere, so the algorithmic manifold is homeomorphic to 2 -sphere from Gauss-Bonnet theorem. When $0<k(c) \leq \frac{1}{2}$ and $v=0$, the Gaussian curvature $K$ of the algorithmic manifold with the metric consisting of equations ( 1) and ( 9) becomes infinite everywhere. At this time, the algorithmic manifold has a vertical tangent as viewed from $v$. From the viewpoint of $\tau$, the Gaussian curvature $K$ of the algorithmic manifold becomes zero everywhere. The algorithmic manifold is also complete, so it is a cylinder from Hartman-Nirenberg theorem [5]. In any case, it can be seen that arbitrary two points on the algorithmic manifold having the metric consisting of the equations (1) and (9) are connected by a geodesic. The existence of a geodesic on an algorithmic manifold means that an algorithm exists. That is, arbitrary calculation problems can set the start and end points of calculation on the algorithmic manifold having the metric consisting of 
equations (1) and (9), so that it turns out those problems have such algorithms the calculation time $\tau$ becomes $\tau=\llbracket v^{k(c)} \rrbracket c$.

Next, in the equation ( 1 ), consider the following $f(v)$. Here, $k(c)$ is a real number variable defined so as to satisfy the relational expression of $\tau$ and $c$.

$$
f(v)=2^{v^{k(c)}}
$$

The Gaussian curvature $K$ of an algorithmic manifold with a metric consisting of equations ( 1 ) and (11) is given by the following equation.

$$
K=0
$$

From equation (12), the algorithmic manifold with the metric consisting of equations (1) and (11) is complete and its Gaussian curvature $K$ is zero everywhere, so the algorithmic manifold is a cylinder from Hartman-Nirenberg theorem [5]. Also in this case, it can be seen that arbitrary two points on the algorithmic manifold having the metric consisting of the equations ( 1 ) and (11) are connected by a geodesic. The existence of a geodesic on an algorithmic manifold means that an algorithm exists. That is, arbitrary calculation problems can set the start and end points of calculation on the algorithmic manifold having the metric consisting of equations ( 1 ) and (11), so that it turns out those problems have such algorithms the calculation time $\tau$ becomes $\tau=\llbracket 2^{v^{k(c)}} \rrbracket c$.

Finally, in the equation ( 1$)$, consider the following $f(v)$. Here, $k(c)$ is a real number variable defined so as to satisfy the relational expression of $\tau$ and $c$.

$$
f(v)=v^{k(c)} \log _{2} v
$$

The Gaussian curvature $K$ of an algorithmic manifold with a metric consisting of equations ( 1 ) and (13) is given by the following equation.

$$
K=\left\{\begin{array}{c}
\frac{1}{v^{2} \log _{2} v}(k(c)=0) \\
\frac{1}{v^{2}}\left(0<k(c) \leq \frac{1}{2}\right) \\
0\left(k(c)>\frac{1}{2}\right)
\end{array}\right.
$$

When $k(c)>\frac{1}{2}$, from equation (14), the algorithmic manifold with the metric consisting of equations ( 1 ) and (13) is complete and its Gaussian curvature $K$ is zero everywhere, so the algorithmic manifold is a cylinder from Hartman-Nirenberg theorem [5]. When $0<k(c) \leq \frac{1}{2}$ and $v>0$, from equation (14), the algorithmic manifold with the metric consisting of equations ( 1 ) and ( 13) is compact and its Gaussian curvature $K$ is positive everywhere, so the algorithmic manifold is homeomorphic to 2 -sphere from Gauss-Bonnet theorem. When $k(c)=0$ and $v>1$, from equation (14), the algorithmic 
manifold with the metric consisting of equations ( 1$)$ and (13) is compact and its Gaussian curvature $K$ is positive everywhere, so the algorithmic manifold is homeomorphic to 2 -sphere from Gauss-Bonnet theorem. When $k(c)=0$ and $0<v<1$, from equation (14), the algorithmic manifold with the metric consisting of equations ( 1 ) and (13) is compact and its Gaussian curvature $K$ is negative everywhere, so the algorithmic manifold is homeomorphic to pseudosphere from Gauss-Bonnet theorem. When $0<k(c) \leq \frac{1}{2}$ and $v=0$, or $k(c)=0$ and $v=0$, or $k(c)=0$ and $v=1$, the Gaussian curvature $K$ of the algorithmic manifold with the metric consisting of equations ( 1 ) and ( 13 ) becomes infinite everywhere. At this time, the algorithmic manifold has a vertical tangent as viewed from $v$. From the viewpoint of $\tau$, the Gaussian curvature $K$ of the algorithmic manifold becomes zero everywhere. The algorithmic manifold is also complete, so it is a cylinder from Hartman-Nirenberg theorem [5]. In any case, it can be seen that arbitrary two points on the algorithmic manifold having the metric consisting of the equations (1) and (13) are connected by a geodesic. The existence of a geodesic on an algorithmic manifold means that an algorithm exists. That is, arbitrary calculation problems can set the start and end points of calculation on the algorithmic manifold having the metric consisting of equations (1) and (13), so that it turns out those problems have such algorithms the calculation time $\tau$ becomes $\tau=\llbracket v^{k(c)} \log _{2} v \rrbracket c$.

3. Relationship between algorithmic manifolds and amounts of data

Think about the algorithmic manifold defined by the following metric.

$$
d s^{2}=d v^{2}+d \tau^{2}=\llbracket m(v)^{2} \rrbracket d w^{2}+d \tau^{2}
$$

Where $w \geq 0$ is amounts of calculation per memory used for calculation, $\llbracket m(v) \rrbracket$ is a function of amounts of data required to process a certain amount of calculation, $v=\llbracket m(v) \rrbracket w$. All variables and functions are real numbers or real functions. Since the algorithmic manifold is a subset of Euclidean space, it is a complete metric space. Therefore, from the Hopf-Rinow theorem [4], there is a geodesic between arbitrary two points. Also, since the algorithmic manifold is a complete metric space, the Cauchy sequence converges. Therefore, since the algorithmic manifold is complete and totally bounded, it is also compact.

In the equation ( 15$)$, consider the following $m(v)$. Here, $k(w)$ is a real number variable defined so as to satisfy the relational expression of $v$ and $w$.

$$
m(v)=v^{k(w)}
$$

The Gaussian curvature $K$ of an algorithmic manifold with a metric consisting of equations (15) and (16) is given by the following equation. 


$$
K=0
$$

From equation (17), the algorithmic manifold with the metric consisting of equations ( 15) and ( 16) is complete and its Gaussian curvature is zero everywhere, so the algorithmic manifold is a cylinder from Hartman-Nirenberg theorem [5]. In this case, it can be seen that arbitrary two points on the algorithmic manifold having the metric consisting of the equations (15) and (16) are connected by a geodesic. The existence of a geodesic on an algorithmic manifold means that an algorithm exists. That is, arbitrary calculation problems can set the start and end points of calculation on the algorithmic manifold having the metric consisting of equations (15) and (16), so that it turns out those problems have such algorithms amounts of data $v$ becomes $v=\llbracket v^{k(w)} \rrbracket w$.

Next, in the equation (15), consider the following $m(v)$. Here, $k(w)$ is a real number variable defined so as to satisfy the relational expression of $v$ and $w$.

$$
m(v)=2^{v^{k(w)}}
$$

The Gaussian curvature $K$ of an algorithmic manifold with a metric consisting of equations (15) and (18) is given by the following equation.

$$
K=0
$$

From equation (19), the algorithmic manifold with the metric consisting of equations ( 15) and ( 18) is complete and its Gaussian curvature is zero everywhere, so the algorithmic manifold is a cylinder from Hartman-Nirenberg theorem [5]. In this case, it can be seen that arbitrary two points on the algorithmic manifold having the metric consisting of the equations (15) and (18) are connected by a geodesic. The existence of a geodesic on an algorithmic manifold means that an algorithm exists. That is, arbitrary calculation problems can set the start and end points of calculation on the algorithmic manifold having the metric consisting of equations (15) and (18), so that it turns out those problems have such algorithms amounts of data $v$ becomes $v=\llbracket 2^{v^{k(w)}} \rrbracket w$.

Finally, in the equation ( 15$)$, consider the following $m(v)$. Here, $k(w)$ is a real number variable defined so as to satisfy the relational expression of $v$ and $w$.

$$
m(v)=v^{k(w)} \log _{2} v
$$

The Gaussian curvature $K$ of an algorithmic manifold with a metric consisting of equations ( 15 ) and ( 20) is given by the following equation.

$$
K=0
$$

From equation (21), the algorithmic manifold with the metric consisting of equations ( 15 ) and ( 20) is complete and its Gaussian curvature is zero everywhere, so the algorithmic manifold is a cylinder from Hartman-Nirenberg theorem [5]. In this case, it can be seen that arbitrary two points on the algorithmic manifold having the metric 
consisting of the equations (15) and (20) are connected by a geodesic. The existence of a geodesic on an algorithmic manifold means that an algorithm exists. That is, arbitrary calculation problems can set the start and end points of calculation on the algorithmic manifold having the metric consisting of equations ( 15) and (20) so that it turns out those problems have such algorithms amounts of data $v$ becomes $v=\llbracket v^{k(w)} \log _{2} v \rrbracket w$.

4. Topological characteristics of algorithmic manifolds

In the section 2, I discussed the case where $\tau=\llbracket v^{k(c)} \rrbracket c$ and $\tau=\llbracket 2^{v^{k(c)}} \rrbracket c$ and $\tau=\llbracket v^{k(c)} \log _{2} v \rrbracket c$, and the Gaussian curvature $K$ of the algorithmic manifold was zero everywhere when $k(c)=0$ or $k(c)>\frac{1}{2}$ or $0<k(c) \leq \frac{1}{2}$ and $v=0$ in the case of $\tau=\llbracket v^{k(c)} \rrbracket c$, and when $k(c)>\frac{1}{2}$ or $0<k(c) \leq \frac{1}{2}$ and $v=0$ or $k(c)=0$ and $v=0$ or $k(c)=0$ and $v=1$ in the case of $\tau=\llbracket v^{k(c)} \log _{2} v \rrbracket c$, regardless of $k(c)$ in the case of $\tau=\llbracket 2^{v^{k(c)}} \rrbracket c$.

In the section 3, I also discussed the case where $v=\llbracket v^{k(w)} \rrbracket w$ and $v=\llbracket 2^{v^{k(w)}} \rrbracket w$ and $v=\llbracket v^{k(w)} \log _{2} v \rrbracket w$, and the Gaussian curvature $K$ of the algorithmic manifold was zero everywhere in each case.

In other words, since the eleven kinds of algorithmic manifolds discussed up to the previous paper are cylinders, it can be said that the eleven kinds of algorithmic manifolds are homeomorphic with each other.

From these facts, it can be said that it is meaningful to discuss and distinguish between characteristic parts and common parts in each of the eleven kinds of algorithms. By increasing the speed of the common parts, speeding up can be achieved in all eleven kinds of algorithms, for example.

Also, in the section 2, when $0<k(c) \leq \frac{1}{2}$ and $v>0$ in the case of $\tau=\llbracket v^{k(c)} \rrbracket c$ and $\tau=\llbracket v^{k(c)} \log _{2} v \rrbracket c$, and when $k(c)=0$ and $v>1$ in the case of $\tau=\llbracket v^{k(c)} \log _{2} v \rrbracket c$, algorithmic manifolds of three cases are homeomorphic to 2 -sphere with each other.

From these facts, also, it can be said that it is meaningful to discuss and distinguish between characteristic parts and common parts in each of the three kinds of algorithms. Also, by increasing the speed of the common parts, speeding up can be achieved in all three kinds of algorithms, for example.

Moreover, when creating a new algorithm, it is possible to judge whether it is homeomorphic with the existing first eleven cases or second three cases or also other cases by checking the Gaussian curvature $K$ of the algorithmic manifold. That is, in 
algorithmic manifolds, the Gaussian curvature $K$ can be said to be one candidate for the indicator.

\section{Conclusion}

I defined the Riemannian manifold with the metric of equation (1) or (15) as the algorithmic manifold and described the properties derived from it.

Firstly, an arbitrary calculation problem was found to have an algorithm in which the calculation time $\tau$ is $\tau=\llbracket v^{k(c)} \rrbracket c$ or $\tau=\llbracket 2^{v^{k(c)}} \rrbracket c$ or $\tau=\llbracket v^{k(c)} \log _{2} v \rrbracket c$. That is, there are algorithms of $\tau=\llbracket v^{k(c)} \rrbracket c$ or $\tau=\llbracket v^{k(c)} \log _{2} v \rrbracket c$ even in calculation problems where the algorithm of $\tau=\llbracket 2^{v^{k(c)}} \rrbracket c$ is currently known. Conversely even in calculation problems where the algorithm of $\tau=\llbracket v^{k(c)} \rrbracket c$ is known, there are algorithms of $\tau=\llbracket 2^{v^{k(c)}} \rrbracket c$ or $\tau=\llbracket v^{k(c)} \log _{2} v \rrbracket c$. Furthermore even in calculation problems where the algorithm of $\tau=\llbracket v^{k(c)} \log _{2} v \rrbracket c$ is known, there are algorithms of $\tau=\llbracket v^{k(c)} \rrbracket c$ or $\tau=\llbracket 2^{v^{k(c)}} \rrbracket c$.

Secondary, an arbitrary calculation problem was found to have an algorithm in which amounts of data $v$ is $v=\llbracket v^{k(w)} \rrbracket w$ or $v=\llbracket 2^{v^{k(w)}} \rrbracket w$ or $v=\llbracket v^{k(w)} \log _{2} v \rrbracket w$. That is, there are algorithms of $v=\llbracket v^{k(w)} \rrbracket w$ or $v=\llbracket v^{k(w)} \log _{2} v \rrbracket w$ even in calculation problems where the algorithm of $v=\llbracket 2^{v^{k(w)}} \rrbracket w$ is currently known. Conversely even in calculation problems where the algorithm of $v=\llbracket v^{k(w)} \rrbracket w$ is known, there are algorithms of $v=\llbracket 2^{v^{k(w)}} \rrbracket w$ or $v=\llbracket v^{k(w)} \log _{2} v \rrbracket w$. Furthermore even in calculation problems where the algorithm of $v=\llbracket v^{k(w)} \log _{2} v \rrbracket w$ is known, there are algorithms of $v=\llbracket v^{k(w)} \rrbracket w$ or $v=\llbracket 2^{v^{k(w)}} \rrbracket w$.

Finally, I discuss the topological properties of algorithmic manifolds and stated their advantages and that the Gaussian curvature $K$ can be raised as candidates for the indicator.

\section{References}

[1] M. Sipser, "Introduction to the Theory of Computation," PWS Publishing, 1997. 
[2] A. A. Razborov , S. Rudich, "Natural Proofs," In Proceedings of the 26th ACM Symposium on Theory of Computing, 1994.

[3] M. A. Nielsen, M. R. Dowling, M. Gu , A. C. Doherty, "Quantum Computation as Geometry,” Science, 2006.

[4] H. Hopf , W. Rinow, "Ueber den Begriff der vollständigen differentialgeometrischen Fläche,” Commentarii Mathematici Helvetici. 3(1), 1931.

[5] P. Hartman , L. Nirenberg, "On Spherical image whose jacobians do not change signs,” Amer. J. Math. 81, 1959. 